\title{
On the $\delta$ Scuti variability in the pre-main sequence Herbig Ae star HR 5999
}

\author{
D. W. Kurtz ${ }^{1,2}$ and C. Catala ${ }^{1}$ \\ 1 Laboratoire d'Astrophysique de l'OMP, CNRS UMR 5572, Observatoire Midi-Pyrénées, \\ 14 avenue Édouard Belin, 31400 Toulouse, France \\ 2 Department of Astronomy, University of Cape Town, Rondebosch 7701, South Africa
}

Received 19 October 2000 / Accepted 26 January 2001

\begin{abstract}
A reanalysis of photometric data for the bright, southern Herbig Ae star HR 5999 obtained by Praderie et al. (1991) at ESO in 1985 shows $\delta$ Scuti pulsation with the same period, $4.812 \mathrm{~d}^{-1}$, and semi-amplitude, $6 \mathrm{mmag}$, found by Kurtz \& Marang (1995) in data obtained from SAAO in 1994. HR 5999 is, therefore, a potential candidate to test Breger \& Pamyatnykh's (1998) predictions of evolutionary period changes 10 to 100 times faster in premain sequence $\delta$ Scuti stars than in post-main sequence stars. We argue that the low frequency, low amplitude $\alpha^{2}$ CVn variability of HR 6000 with a period near $2 \mathrm{~d}$, and its long-term variability are no hindrance to using it as a comparison star for study of the $\delta$ Scuti variability of HR 5999. With a separation of 44 arcsec between HR 5999 and HR 6000, and similar brightnesses for the two stars, we urge the use of small telescopes with CCD photometers to obtain the long-term data necessary for the study of period change. We point out that this can be done under less-than-ideal photometric conditions and urban lighting with CCD photometers on small telescopes which are widely available at small observatories, at universities and at the home observatories of serious amateur astronomers.
\end{abstract}

Key words. stars: circumstellar matter - stars: HR5999 - stars: oscillations: pre-main sequence: variables: $\delta$ Sct

\section{Introduction}

The Herbig Ae/Be stars are pre-main sequence objects with masses ranging from 2 to $5 M_{\odot}$. A very significant fraction of them show conspicuous signs of strong stellar winds and of chromospheric activity. Many of them also show non-periodic photometric variability from variations in the transparency of circumstellar dust. This nonperiodic variability can exceed $1 \mathrm{mag}$, yet recently seven Herbig Ae stars which lie within the instability strip have been found to be $\delta$ Scuti pulsators (HR 5999 - Kurtz \& Marang 1995; HD 104237 - Donati et al. 1997; Kurtz \& Müller 1999; HD 142666 - Kurtz \& Müller 2001; HD 35929 and V351 Ori - Marconi et al. 2000; BL 50 and HP 57 in NGC 6823 - Pigulski et al. 2000), even though their pulsation amplitudes are less than a few hundredths of a mag. There are also two pre-main sequence stars (not classified as Herbig Ae stars) in NGC 2264 which are known to be $\delta$ Scuti stars, V588 Mon (HD 261331; Walker 2) and V599 Mon (Walker 20) (Breger 1972). It is now clear that the time-scales for the long-term, non-periodic photometric variations and short-period $\delta$ Scuti pulsations are

Send offprint requests to: D. W. Kurtz, e-mail: kurtz@ast.obs-mip.fr well-separated in frequency space so that Fourier analysis of the $\delta$ Scuti pulsations alone is feasible.

Asteroseismology of pre-main sequence $\delta$ Scuti stars has the potential to provide unprecedented constraints on models of these stars, and it has the possibility of detecting evolutionary period changes, thus providing a direct measure of the pre-main sequence evolutionary time-scale. Breger \& Pamyatnykh (1998) estimated that the rate of period change for pre-main sequence $\delta$ Scuti stars is 10 to 100 times faster than for post-main sequence stars - up to $(1 / P) \mathrm{d} P / \mathrm{d} t=10^{-6} \mathrm{yr}^{-1}$. This is similar to the precision possible in the measurement of the frequencies of low-amplitude $\delta$ Scuti stars in a few years of observation. Thus far, only discovery light curves are available for the few known pre-main sequence pulsators, so that it is not known if their modes are stable, if the stars are multiperiodic like many post-main sequence $\delta$ Scuti stars, or what their frequency distributions look like. The discovery of more of these stars and the intensive study of the frequencies of selected objects are highly desirable.

The brightest such star, and arguably the best candidate for a long-term study of its pulsation behaviour, is the southern star HR 5999 (HD 144668; V856 Sco) - a pre-main sequence Herbig A7 III-IVe star that has been intensively studied at many wavelengths for decades. 
The literature on this star is extensive and most general discussions of Herbig Ae/Be stars make some reference to it. Photometrically, HR 5999 was studied by Baade \& Stahl (1989), Praderie et al. (1991) and Pérez et al. (1992), the latter using data from the "Longterm Photometry of Variables at ESO" project for which the latest reference is Sterken et al. (1993). Pérez et al. (1992) showed that HR 5999 varies from a maximum brightness of $V \approx 6.8$ down to (occasionally) $V>8$. Although it undergoes continuous irregular photometric variability, most of the time it is within $0.5 \mathrm{mag}$ of its maximum brightness. None of the above studies was attempting to discover $\delta$ Scuti pulsation, as it was not expected at the time.

HR 5999 lies in the $\delta$ Scuti instability strip (see Marconi \& Palla 1998). It is a fast rotator with $v \sin i=$ $180 \pm 50 \mathrm{~km} \mathrm{~s}^{-1}$, estimated mass of $M=3 M_{\odot}$, radius of $R=6.9 R_{\odot}$, temperature of $T_{\text {eff }} \approx 7800 \mathrm{~K}$ and surface gravity $\log g \approx 3.5-4.0$ (Böhm \& Catala 1995; Tjin a Djie et al. 1989). Kurtz \& Marang (1995) found HR 5999 to be a $\delta$ Scuti star with a period of about $5 \mathrm{~h}$ and a peakto-peak amplitude in Johnson $V$ of 0.013 mag. HR 5999 is probably associated physically with the peculiar late B star HR 6000 which is separated from it by 44 arcsec.

Kurtz \& Marang showed that HR 6000 is an $\alpha^{2}$ CVn variable with a period of about $2 \mathrm{~d}$. Small amplitude variations with a time-scale of years in Strömgren $u v b$ (but not $y$ ) were found by van den Ancker et al. (1996) which they proposed may be caused by a $\mathrm{T}$ Tauri companion. This 2-d periodic variability of HR 6000, any long-term variability in it or a companion star and the long-term, large amplitude erratic variability of HR 5999 itself are sufficiently well-separated in frequency from the 5 -h periodicity of HR 5999 that its $\delta$ Scuti pulsation can be studied - even using HR 6000 as the comparison star.

Following the discovery of the pulsation in HR 5999, a re-examination of the light curve data plotted in Fig. 5 of Praderie et al. (1991) suggested that the $\delta$ Scuti variability might be present in those data, if only they were examined at higher resolution and with Fourier analysis. Although those data were obtained with the intention of studying the long-term variations caused by variable circumstellar dust, they have sufficient time resolution to test for $\delta$ Scuti pulsation. We therefore decided to re-analyse them with $\delta$ Scuti variability in mind. In this paper we show that the pulsation is present in Praderie et al. (1991) data with the same period and amplitude found by Kurtz \& Marang (1995).

\section{Analysis and results}

Photometric observations of HR 5999 through Johnson $U B V R I$ filters were obtained on six consecutive nights, 4 to 9 March 1985, using the ESO 0.5-m telescope. HR 6000 was observed as a comparison star for differential photometry, since neither its low amplitude $(<0.01 \mathrm{mag}) 2-\mathrm{d}$ variability (Kurtz \& Marang 1995), nor its low-amplitude, long-term variability (van den Ancker et al. 1996) were known at that time. See Praderie et al. (1991) for more details. See, especially, their Fig. 5 which is a low-time resolution light curve of the $V$ data. The data were gathered at a sampling rate of about once every $9 \mathrm{~min}$, so that they cover the 5-h pulsation of HR 5999 well. Other published photometric data, such as those in the "Longterm photometry of variables at ESO" project (Sterken et al. 1993) are sampled usually once per night, an interval too long to test for $\delta$ Scuti pulsation.

For the purpose of testing for very low amplitude pulsation, only high precision photometry can be used. An examination of the Praderie et al. data shows that their first three nights (hereafter: nights 1,2 and 3) and last night (night 6) are of sufficient quality for our purposes here. Night 4 was not photometric and only three data points were obtained; night 5 was not sufficiently photometric to be included in our analysis. The caption to Fig. 5 of Praderie et al. notes the poor photometric conditions on nights 4 and 5, and the error bars in their Fig. 5 confirm this. Since only one comparison star is available, HR 6000, the photometric precision cannot be measured by differential photometry of two comparison stars. The absolute photometry of HR 6000, however, gives a quantitative measure of the quality of the nights. The standard deviation per observation for the HR 6000 magnitudes transformed to the standard system using an observed set of standard stars (see Praderie et al. for details) gives the following values: $\sigma_{V}=0.005 \mathrm{mag}$ (night 1 ); $\sigma_{V}=0.013 \mathrm{mag}$ (night 2); $\sigma_{V}=0.010 \mathrm{mag}$ (night 3 ); $\sigma_{V}=0.070 \mathrm{mag}$ (night 5); and $\sigma_{V}=0.003 \mathrm{mag}$ (night 6 ). These numbers confirm that night 5 could not be included in our analysis, and that nights 1, 2, 3 and 6 were of good to excellent photometric quality. It must be remembered that the standard deviations given above are not differential, hence the differential precisions expected for the HR 5999 data compared to the HR 6000 data are better than this and are of the order of several thousandths of a mag; our frequency analysis below confirms this and shows the average standard deviation per observation for the $V$ data to be $\sigma=4.5$ mmag.

Originally, the Praderie et al. data were reduced differentially with respect to HR 6000 by simple subtraction of pairs of magnitudes obtained during the same observing cycle of variable-comparison-sky. This was reasonable, since in each cycle HR 5999 and HR 6000 magnitudes were separated by only about 3 to $4 \mathrm{~min}$ and large variations were being studied. However, we re-reduced the data differentially by time-interpolating the HR 6000 magnitudes to the times of observation of the HR 5999 magnitudes, thus improving the differential photometric precision further, as is necessary for the study of low amplitude pulsation.

We then performed a Fourier analysis of the ESO $U B V R I$ data for each colour separately in the same manner as that done by Kurtz \& Marang (1995) for the SAAO data. First, we removed the mean magnitude for each night to filter out some of the long-term, high-amplitude, non-periodic variability caused by the variable circumstellar dust. This does not fully model the low-frequency 

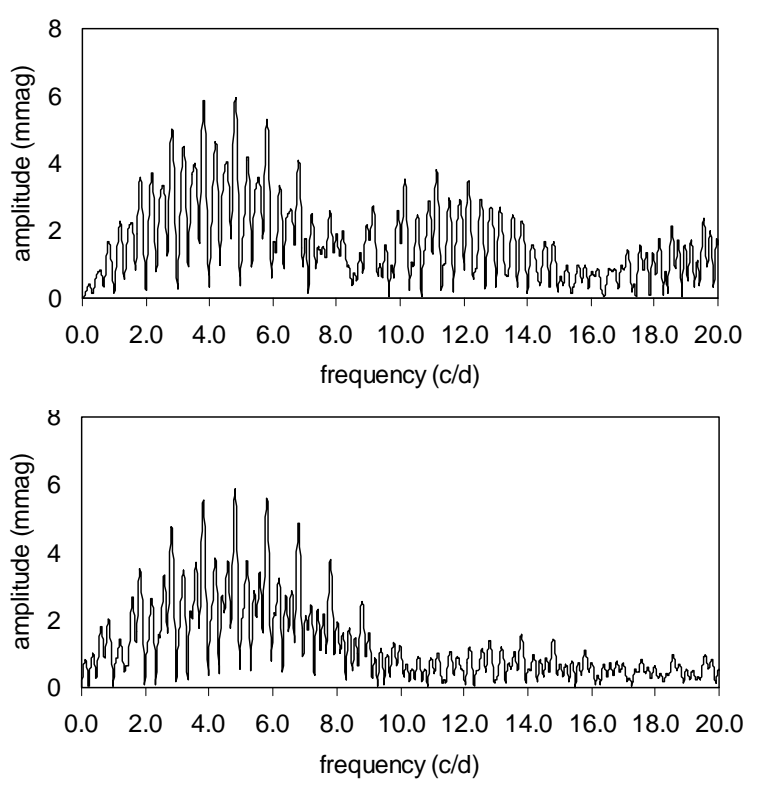

Fig. 1. Top panel: The amplitude spectrum of the Praderie et al. (1991) $V$ data. Bottom panel: The spectral window for those data. The highest peak at $4.812 \mathrm{~d}^{-1}$ is identical with the highest peak in the amplitude spectrum of the Kurtz \& Marang (1995) $V$ data shown in Fig. 2. The spectral window here and that in Fig. 2 were generated with an artificial, noisefree sine wave sampled at exactly the times of the observations with the frequency and amplitude of the highest peak in the actual amplitude spectrum

variations, but is the best that can be done with the relatively short data sets available. We then Fourier transformed the data using a Discrete Fourier Transform for unequally spaced data (Deeming 1975; Kurtz 1985). We show the amplitude spectrum of the Praderie et al. $V$ data in the top panel of Fig. 1 and, at exactly the same scale, the amplitude spectrum of the Kurtz \& Marang data in the top panel of Fig. 2. Both have a highest peak at $4.812 \mathrm{~d}^{-1}$. A linear least squares fit of that frequency to the data gives an amplitude of $5.6 \pm 0.7 \mathrm{mmag}$ for the Praderie et al. data and $6.8 \pm 0.2 \mathrm{mmag}$ for the Kurtz \& Marang data. The difference between these, $1.2 \pm 0.7 \mathrm{mmag}$ is not significant, indicating that the same frequency is present at the same amplitude in both data sets separated by 9 yr.

However, we are not completely confident that we have selected the correct alias. In both Figs. 1 and 2 the noise level is higher than the difference in amplitude between the highest peak and its nearest $1 \mathrm{~d}^{-1}$ aliases, making the correct choice uncertain. To look at this problem further we performed identical analyses on the data in each colour for the ESO data. Figure 3 shows the amplitude spectra for all five colours, $U B V R I$. The $U, R$ and $I$ data have a highest peak at the same frequency as the $V$ data; the $B$ data have a peak there, although the highest peak is at a lower frequency. We conclude that all of the data support the identification of the $V$ frequency, but that the available signal-to-noise ratio and alias structure do not allow certain identification of the correct alias. This was true of the Kurtz \& Marang study also. That the
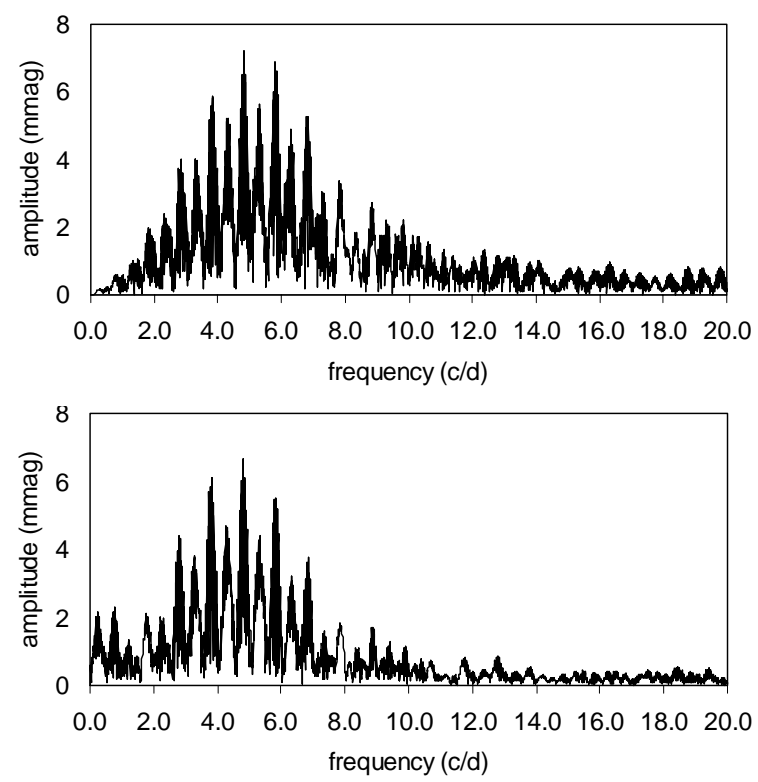

Fig. 2. Top panel: The amplitude spectrum of the Kurtz \& Marang (1995) $V$ data. Bottom panel: the spectral window for those data. See the caption of Fig. 1 for more information

Praderie et al. $V$ data and the Kurtz \& Marang $V$ data find the same frequency, indicates that it is probably the right alias, but only better data will resolve this. This is obviously important, since astrophysical inference, such as mode identification and estimates of density and luminosity as attempted by Kurtz \& Marang, are based on the pulsation period.

Least-squares fits of the frequency $4.812 \mathrm{~d}^{-1}$ to the $U B V R I$ data give the amplitudes and standard deviations of the residuals shown in Table 1 . The last column gives the standard deviation per observation for the residuals to the fitted $4.812-\mathrm{d}^{-1}$ frequency. These are excellent estimates of the precision of the differential observations, and it is the result for the $V$ data that we previously used to support our estimate of the precision. Table 1 also shows that the amplitudes in $B$ and $V$ are not significantly different, given our errors, so that application of mode identification techniques using amplitude ratios and phase differences (cf. Watson 1988) is not possible with these data.

To test for multi-periodicity (which is common in $\delta$ Scuti stars) we prewhitened both data sets in the same manner. Figure 4 shows the amplitude spectra of the residuals of the Praderie et al. (1991) and Kurtz \& Marang (1995) $V$ data (top and bottom panels, respectively) plotted on the same scale. The two plots show no similarities so that no definitive conclusion can be reached about the possible presence of any other pulsation frequencies. After fitting the $4.812 \mathrm{~d}^{-1}$ frequency to each data set the standard deviations of the residuals are consistent with our estimate of the quality of the data. 

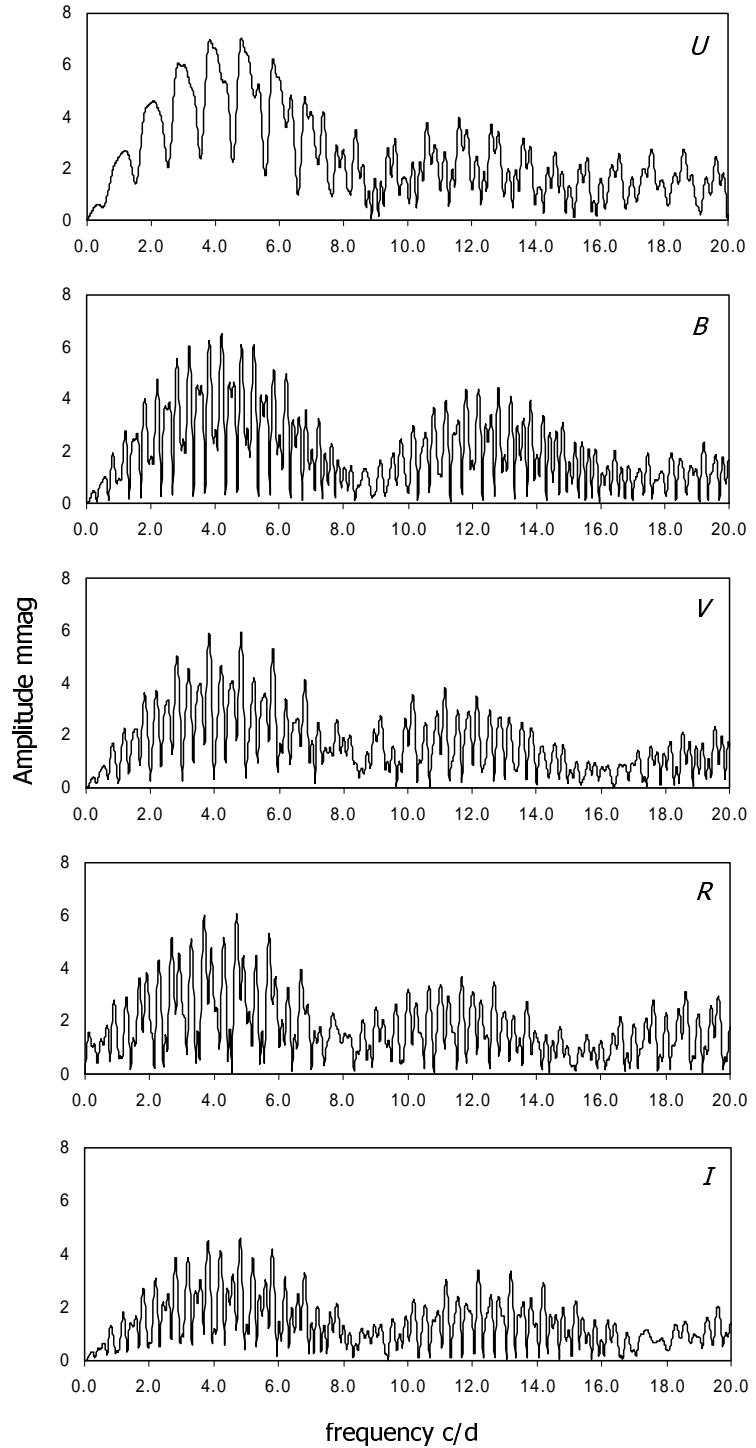

Fig. 3. The amplitude spectra of the $U B V R I$ ESO data. The data in all colours support the identification of the highest peak in the $V$ data, but cannot definitely determine the correct alias. The apparently poorer frequency resolution of the $U$ amplitude spectrum is caused by noise in the data producing unresolved, closely-spaced peaks

\section{Prospects for the study of the $\delta$ Scuti pulsation in HR 5999}

We have shown that HR 5999 was pulsating in the same mode in 1985 and 1994. This makes HR 5999 astrophysically a prospect for the study of evolutionary period change in a pre-main sequence $\delta$ Scuti star. Breger \& Pamyatnykh's (1998) estimated a rapid rate of evolutionary period change of up to $(1 / P) \mathrm{d} P / \mathrm{d} t=10^{-6} \mathrm{yr}^{-1}$ for pre-main sequence $\delta$ Scuti stars. This is the level of precision obtained by Handler et al. (1998) for the third frequency in the $\delta$ Scuti star XX Pyx which has a similar small amplitude to that presented here for HR 5999 with observations spanning $4 \mathrm{yr}$. Hence the possibility exists that an evolutionary period change could be measured in
Table 1. Least-squares fits of the frequency $4.812 \mathrm{~d}^{-1}$ to the Praderie et al. (1991) ESO UBVRI data and the Kurtz \& Marang (1995) SAAO $V$ data. Phases are shown for the ESO data to compare them as a function of colour (the zero point for these phases is 0UT 4 March 1985). The phase for the SAAO data is with respect to HDJ2469425.0, but the SAAO and ESO data are separated by too much time to compare the $V$ phases for the two data sets meaningfully

\begin{tabular}{lllll}
\hline filter & observatory & $\begin{array}{l}\text { amplitude } \\
\text { mmag }\end{array}$ & $\begin{array}{l}\text { phase } \\
\text { radians }\end{array}$ & $\begin{array}{l}\sigma \\
\text { mmag }\end{array}$ \\
\hline$U$ & ESO & $7.5 \pm 1.2$ & $-3.57 \pm 0.17$ & 8.0 \\
$B$ & ESO & $5.7 \pm 0.9$ & $-2.97 \pm 0.17$ & 6.0 \\
$V$ & ESO & $5.6 \pm 0.7$ & $-3.10 \pm 0.13$ & 4.5 \\
$R$ & ESO & $2.6 \pm 1.0$ & $-3.08 \pm 0.39$ & 6.3 \\
$I$ & ESO & $4.3 \pm 0.6$ & $-2.94 \pm 0.16$ & 4.2 \\
& & & & \\
$V$ & SAAO & $6.8 \pm 0.2$ & $-1.25 \pm 0.04$ & 2.9 \\
\hline
\end{tabular}
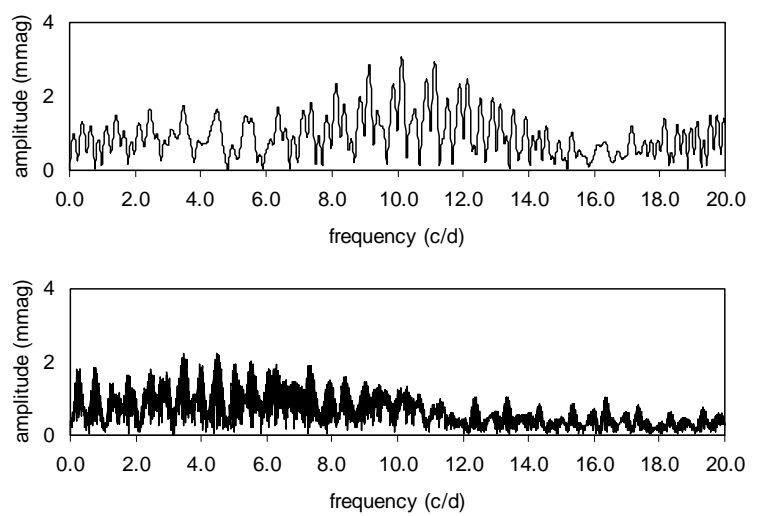

Fig. 4. Top panel: The amplitude spectrum of the Praderie et al. (1991) $V$ data after prewhitening by the highest peak at $4.812 \mathrm{~d}^{-1}$. Bottom panel: the amplitude spectrum of the Kurtz \& Marang (1995) $V$ data after prewhitening by the highest peak at $4.812 \mathrm{~d}^{-1}$. There is no similarity in the two, hence no evidence of a second pulsation frequency at the precision of the data presented here

HR 5999 with a regular program monitoring its light variations over a time-span of 5 to 10 years. As Breger \& Pamyatnykh pointed out, the size of the expected change should make it detectable, even in the presence of other (unexplained) variability in frequency of the type often seen in $\delta$ Scuti stars.

Observations spanning a decade for HR 5999 are feasible. This is because HR 5999 is astronomically a particularly enticing prospect, too: HR 6000 is separated from it by only 44 arcsec and is of similar brightness; the 2.028-d and long-term variability of HR 6000 are well-separated in frequency from the $\delta$ Scuti variability of HR 5999 so that it is no problem in separating the two in Fourier space (in practice the variability of HR 6000 is filtered in the process of removing the long-term variations in HR 5999). This combination means that these stars can be observed with small telescopes equipped with CCD photometers. Since the program star and comparison star are in the same field, good precision can be obtained under 
less-than-photometric conditions. Frandsen et al. (1995) showed this when they obtained an acceptable differential photometric precision for the study of the $\delta$ Scuti star $\kappa^{2}$ Boo with respect to $\kappa^{1}$ Boo using the Ole R $\varnothing$ mer Observatory $50-\mathrm{cm}$ telescope, essentially at sea-level on the outskirts of Århus in Denmark under conditions not normally considered to be photometric. Hence HR 5999 could be observed intensively with the kind of telescope and photometer widely available at small observatories, at universities and, increasing, at the homes of serious amateur astronomers. Even city lights are not a barrier given the brightnesses of HR 5999 and HR 6000. An added bonus of a long-term intensive study of the $\delta$ Scuti frequencies in HR 5999 in this way would be a detailed record of the variability caused by the circumstellar dust.

\section{References}

Baade, D., \& Stahl, O. 1989, A\&A, 209, 255

Böhm, T., \& Catala, C. 1995, A\&A, 301, 155

Breger, M. 1972, ApJ, 171, 539

Breger, M., \& Pamyatnykh, A. 1998, A\&A, 332, 958
Deeming, T. J. 1975, Ap\&SS, 36, 137

Donati, J.-F., Semel, M., Carter, B. D., Rees, D. E., \& Cameron, A. C. 1997, A\&A, 291, 658

Frandsen, S., Jones, A., Kjeldsen, H., et al. 1995, A\&A, 301, 123

Handler, G., Pamyatnykh, A. A., Zima, W., et al. 1998, MNRAS, 295, 377

Kurtz, D. W. 1985, MNRAS, 213, 773

Kurtz, D. W., \& Marang, F. 1995, MNRAS, 276, 191

Kurtz, D. W., \& Müller, M. 1999, MNRAS, 310, 1071

Kurtz, D. W., \& Müller, M. 2001, MNRAS, in press

Marconi, M., \& Palla, F. 1998, ApJ, 507, L141

Marconi, M., Ripepi, V., Alcalà, J. M., et al. 2000, A\&A, 355, 35

Pérez, M. R., Webb, J. R., \& Thé, P. S. 1992, A\&A, 257, 209

Pigulski, A., Kołaczkowski, Z., \& Kopacki, G. 2000, Acta Astron., 50, 113

Praderie, F., Catala, C., Czarny, J., Thé, P. S., \& Tjin a Djie, H. R. E. 1991, A\&AS, 89, 91

Sterken, C., Manfroid, J., Anton, K., et al. 1993, A\&AS, 102, 79

van den Ancker, M. E., de Winter, D., \& Thé, P. S. 1996, A\&A, 313,517

Watson, R. D. 1988, Ap\&SS, 140, 255 